\title{
Research on Problems of the Loss of Distributed Power Grid Switch and Heat Dissipation
}

\author{
Rui Guo ${ }^{1}$, Liaoyi Ning ${ }^{2}$ and Jinyuan Liu $^{2}$ \\ 1School of Renewable Energy, Shenyang Institute of Engineering, Shenyang, \\ China \\ 2Economic Technology Research Institute, State Grid Liaoning Electric Power \\ Supply Co. Ltd, Shenyang, China \\ Email:gr089@sina.com
}

\begin{abstract}
At present, the technology of the distributed power grid gradually become mature. The focus of the study is from controlling power quality to reducing the loss of power grid, improving the network efficiency and reducing the cost. In the link of distributed generation grid connected power generation, the loss of the switching device is an important factor affecting the efficiency of grid connection. This paper puts forward a systematic solution to this problem. Firstly, as for topology structure of grid connected controller, aiming at the research on NPC, a traditional topology which is energy-saving and low loss, we present an improved NPC topology with a more continuous flow state. At the same time for this topology, the corresponding control strategies are designed in this paper. It makes the switching device of the grid connected controller have more freedom of switch and make the system can achieve the precise control of the switch tube, which can achieve the balance of system device lose heat so as to solve the problem of local high temperature from inverter. The system is verified by grid connected test, the power adjustable PWM strategy can be used to achieve different working states without affecting the output current of the system. Through the comparison between light and heat sensors finds that the topology and control strategy proposed in this paper are successfully completed by the new hardware topology and control strategy to achieve the loss balance of the system switching devices.
\end{abstract}

Keywords: grid - connected photovoltaic system, topology structure, loss distribution, control strategy

\section{Introduction}

With the development of new energy industry, the distributed generate on and grid connected technology are also mature. At present, how to reduce the loss of the grid connected distributed generation, improve the efficiency and reduce the cost of power generation has become the focus of research in this field. The loss caused by the switching device of the distributed generation and grid connected controller is an important factor affecting the efficiency of the grid connected. In recent years, one of the main researches for the application of NPC topology is to reduce the loss.

NPC topology has good EMI characteristics. It is firstly applied to the power transmission in the electrical field for eliminating the leakage current caused by the high frequency modulation in the motor, thereby reducing the power loss. At the same time, due to the motor control system and the photovoltaic control system in the topology, the stator and rotor of the leakage capacitance between the solar panel and the capacitance structure is similar to the ground, in recent years, the NPC topology is introduced into the grid connected photovoltaic technology. The traditional NPC topology has three levels of output voltage, similar to unipolar tone $\mathrm{H}$ full bridge, it can improve the inverter 
efficiency and reduce the switching loss. EMI performance is good, but the traditional NPC topology DC input voltage is improved a lot compared to the $\mathrm{H}$ full bridge structure, and switching device loss distribution is easy to cause local overheating of the control system.

In this paper, according to the topology efficiency experiment of grid connected controller, it is obvious that under different conditions, the loss of the devices on different semiconductor devices is significantly different in the traditional NPC topology, the uneven distribution of the device losses will increase the difficulty of the design of the radiator. In view of the above problems, this paper puts forward the improved NPC Active topology and the grid connected PWM strategy. The device loss distribution of the NPC Active topology is balanced by using this PWM strategy and the error free tracking of the sinusoidal current command can be made. Besides, we can get the experimental verification.

\section{Improved NPC Topology}

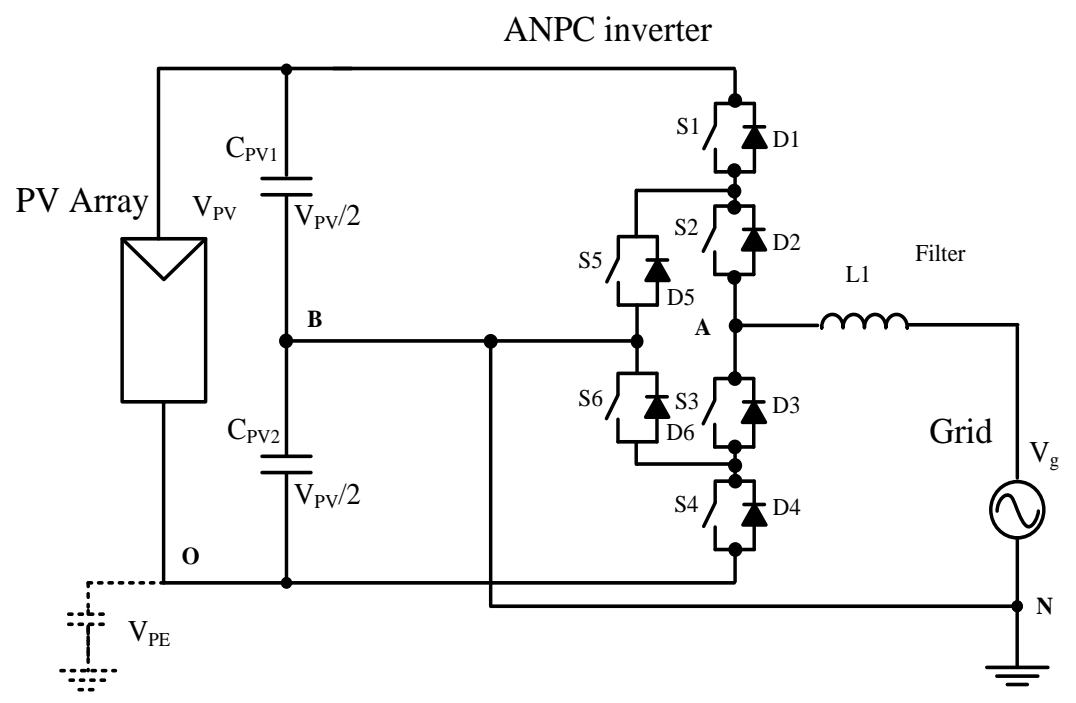

Figure 1. NPC Topology

In this paper, the new NPC topology is shown in Figure 1.In the traditional NPC topology, there is only one continuous flow loop in the current of the different flow direction. Compared with the traditional NPC structure, the proposed topology can have more zero current state in the control process.

As shown in the graph, there are two controllable switching devices in the inverter circuit and the continued flow direction of two to two. Therefore, the corresponding control strategy is provided with a larger free control space to achieve the loss balance of the device.

In this paper, the new topology output voltage waveform has three levels: -VPV/2, VPV/2, 0; Similar to unipolar modulation $\mathrm{H}$ full bridge topology. Compared with the bipolar modulated $\mathrm{H}$ full bridge, the inductance can be reduced and the efficiency of the inverter can be improved. Low switching loss, good performance of EMI;

Compared to the traditional NPC, adding two switching devices (a bridge arm), due to the design of the converter, the use of the same device is conducive to spare parts, At the same time, the effect of adding a switch device bridge on the converter cost is not obvious.

But compared to the traditional NPC topology, theoretically, NPC Active can control the loss distribution of the controller. So NPC Active has more advantages compared to 
the traditional Active; NPC is more suitable for the converter topology with higher power level.

\section{PWM Control Strategy}

According to the topological structure of this paper, we have studied and developed the corresponding control strategy under different working conditions, which can adjust the loss of switching devices and achieve the balance of loss.

Shown in Figure 1, this control strategy can have six continuous flow paths and can complete through application of controlled continuous flow paths by the corresponding PWM strategy to complete loss balance control. Table 1 is a given device switch table.

Table. 1. The Switching States of Strategy

\begin{tabular}{l|rrrrrr}
\hline Voltage & S & S & S & S & S & S \\
& 1 & 2 & 3 & 4 & 5 & 6 \\
\hline Positive & 1 & 1 & 0 & 0 & 0 & 1 \\
0+In & 1 & 0 & 1 & 0 & 0 & 1 \\
0+Out & 0 & 1 & 1 & 0 & 0 & 1 \\
0+ & & & & & & \\
$0-$ & 0 & 0 & 1 & 0 & 0 & 1 \\
0-Out & 0 & 1 & 1 & 0 & 1 & 0 \\
0-In & 0 & 1 & 0 & 1 & 1 & 0 \\
Negative & 0 & 0 & 1 & 1 & 1 & 0 \\
& & & & & & \\
\hline
\end{tabular}

The specific PWM control strategy does not require all of the continued flow path work, in normal operation, only four work paths are needed to complete the control. The periodic variation of six paths switching options is as shown in Figure 2.When system is in the state of commutation of positive half cycle 2 (a) $0+, 0+\operatorname{In}$, positive signal, $0+\operatorname{In}, 0+$ negative half cycle Figure 2 (c) in 0-, 0-In, negative signal, 0-In, 0-, the loss of switching device is on the inner tube. When the system is in the positive half cycle of the commutation order in Figure 2 (b), 0+, 0+Out, positive signal, 0+Out, 0+ negative half cycle 2 (d) 0 -, 0 -Out, negative signal, 0 -Out, 0 -,the loss of switching is reflected in the outer tube.

Figure 2 clearly reflects the process that control strategy of the system is to realize the active state to zero current state: When selecting the $0+$ In over to the $0+$ system switch device continued to flow, at this point the tube S2 is turn off, while the role of the internal tube is off loss. When the 0+Out to 0+ control system continues to flow through the control strategy, the outer tube S1 begin to turn off the power switch off at the same time. By this control strategy, the new NPC topology is proposed in different working states in this paper. According to the programming control switch device, the ideal zero state current circuit is selected to achieve the balance of the switching loss. 

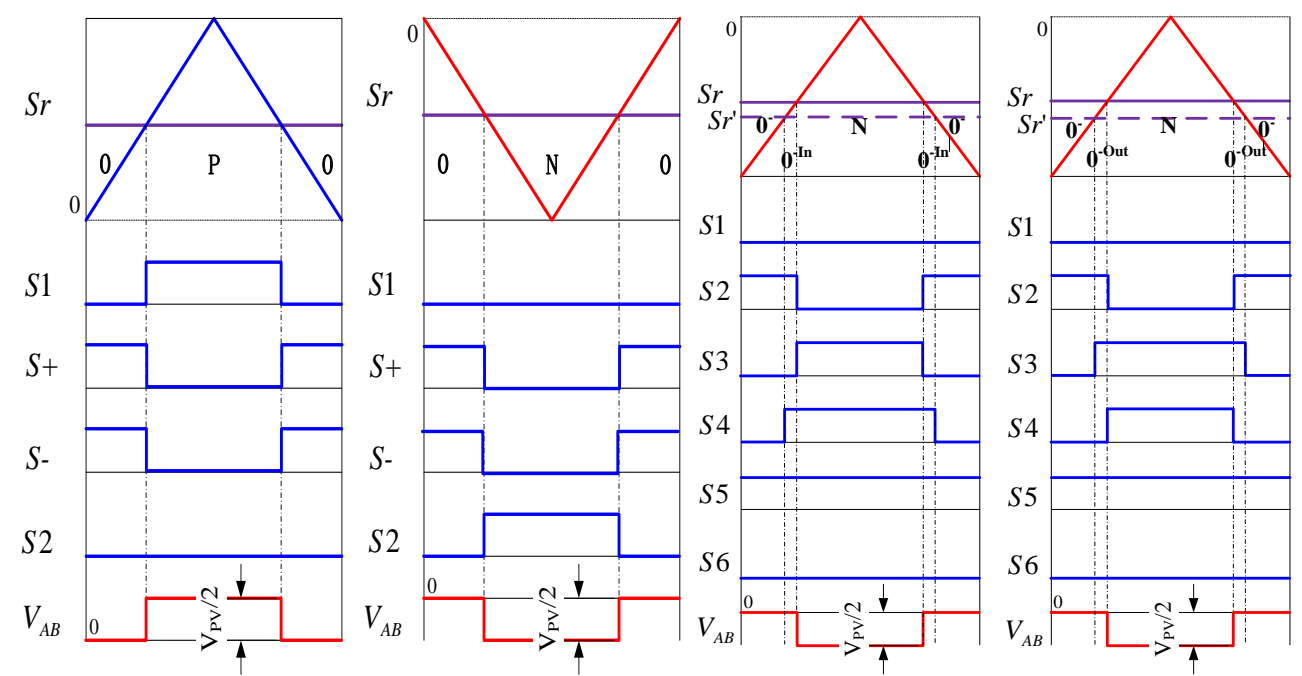

Figure 2. Switching Chart of PWM Strategy

As shown in Figure 2, the switching strategy is to give the original modulated wave signal to multiply a magnification factor $\mathrm{K}$ to form an amplified modulated wave $\mathrm{Sr}$ ' that is the modulation method of PWM control strategy proposed in this paper. In the Stress In state, S1, S4 use triangular carrier and amplified modulation wave Sr' compared to produce PWM control signal; In the Stress Out state, the modulated wave Sr' is working in the positive half cycle of S2, working in the negative half cycle of S3; When the power factor of the system is 1, the S2 and S3 simultaneously use the triangular carrier and the amplified modulation wave Sr' compared to produce the PWM control signal.

Aiming at the control problems arising from the control strategy of the traditional NPC structure, the control methods used in this paper are effective to deal with. As for the problem of output current direction of amplified modulated wave firstly, after research and experiment, this paper find that the output current depends on the active state of the system cycle and the time of the current state, as long as the time control strategy does not change, the system will not amplify the output current due to the modulation wave amplification problem. Whether the output current changed by the use of the amplified modulation wave $\mathrm{Sr}$ 'is shown in Figure 2.When the system is in the active state system $\mathrm{S} 1$, while the S2 is conducting, the system does not increase the modulation wave of the internal and external switch tube at the same time, therefore, the system does not have the output current reverse due to the amplification of the modulation wave. Specific experimental waveforms can refer to Figure 4.

For the problem of choice of system amplification factor, after research ,this paper comes to the conclusion that the amplification factor cannot be set too small, or will cause the transition time too short, the system cannot be completed. At the same time, the modulation amplitude of the system is not more than the amplitude of the carrier by specific experiment, the system will occasionally appear error pulse in this case, the specific reason analysis and the sampling period are related, and need to be further studied.

When setting the Stress In and Stress Out state, the principle of system control strategy is the first choice to consider the device loss balance between inner and outer tubes. Secondly, the loss balance between the two switches is considered with the existence of internal and external tubes.

For the control system, the control strategy adopted in this paper is that when the positive half cycle of S5 and S4 is disconnected and the negative half cycle of S1 and S6 is disconnected, the dead zone is added to the zero crossing point of the power network; 
When the system is in other states, the dead time is not set so that the system cannot be disturbed by the dead time in the state of zero sequence current.

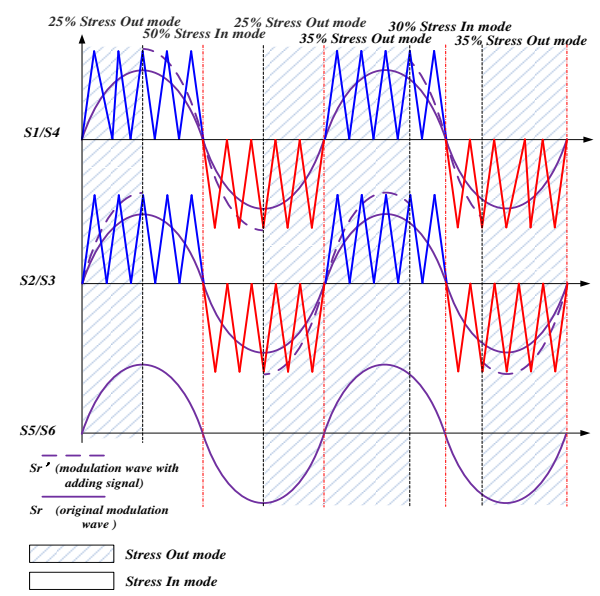

Figure 3. The Work Mode Choice of ALD Active NPC PWM Strategy

As shown in Figure 3, the PWM modulation waveforms of the grid period are shown, in the state of Stress Out/Stress In 50\%-50\%, the inner and outer tubes are under the control of the loss of 50\%; Under the condition of Stress Out/Stress In 70\%-30\%, the loss of inner tube $30 \%$ and outer tube $70 \%$ is realized. At the same time as shown in Figure 3, the dashed line in the diagram is used for the system using modulated wave under different working conditions. Figure 4 is the system's working waveform for the system under the conditions of the power factor of 1.The experimental waveforms of the system can be seen that the system works under different control strategies, and the output current waveform is not affected.

\section{Experiment}

(a) Experimental Waveforms of Stress Out/Stress in 50\%-50\% Mode 
Figure 4. NPC PWM Experimental Waveforms

In this paper, eZDSP28335 is used as the system control core to set up the experimental platform, the experimental results shown in Figure 3 are in the active state, while the inner and outer tubes are simultaneously conducting. The experimental results show that the modulation wave of the inner and outer tubes at different times cannot change the output current. Therefore the control strategy proposed in this paper can achieve the control of switching device loss.

In the experiment of power loss distribution, the system is based on the absence of a heat sink, and the switching frequency of the system is set to $18 \mathrm{kHz}$. The experimental parameters are shown in Table 2:

Table 2. Experimental Parameters

\begin{tabular}{l|ll}
\hline Quantity & Value & Comment \\
\hline AC frequency & $50 \mathrm{~Hz}$ & \\
Switching frequency & $18 \mathrm{kHz}$ & \\
Output inductor L & $6 \mathrm{mH}$ & \\
& &
\end{tabular}




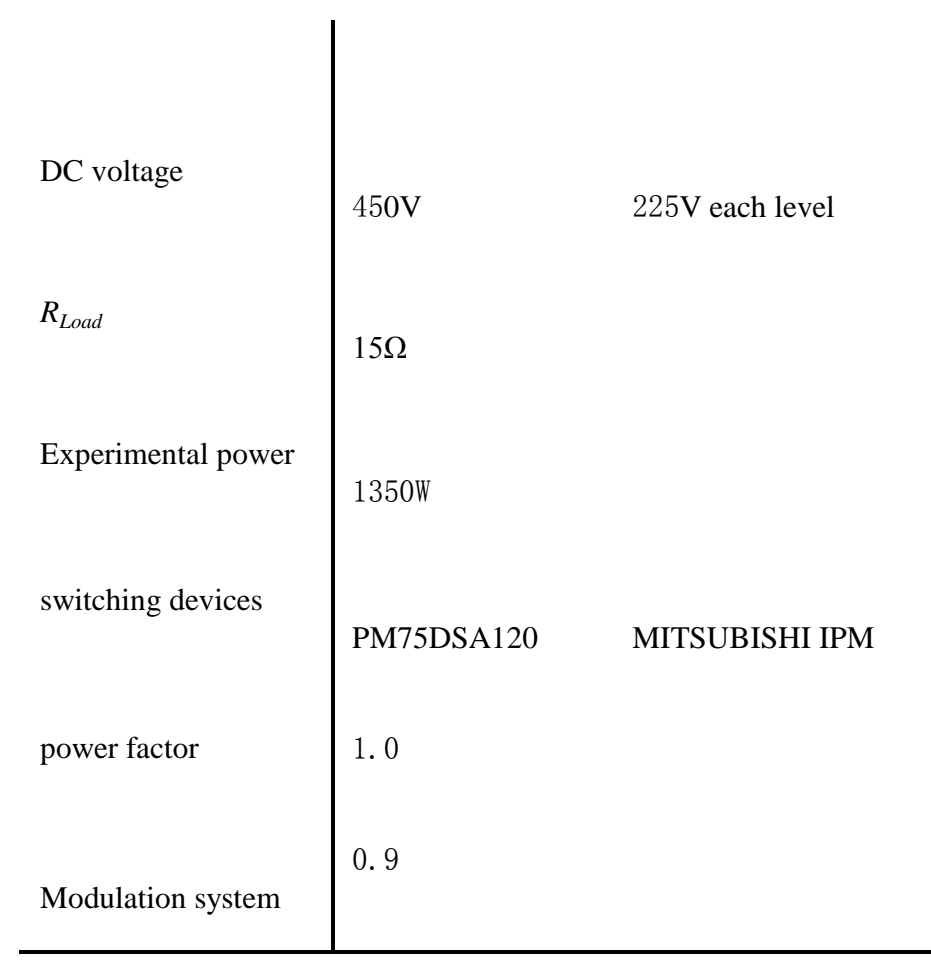

System experimental results are demonstrated by a thermal imager, as shown in figure (b) using 50\%-50\% modulation ratio, inner tube temperature rise rapidly, bear the device loss; As shown in figure (b), in the $80 \%-20 \%$ modulation mode, all the switching devices of the system assume the loss of balance, the temperature is stable.

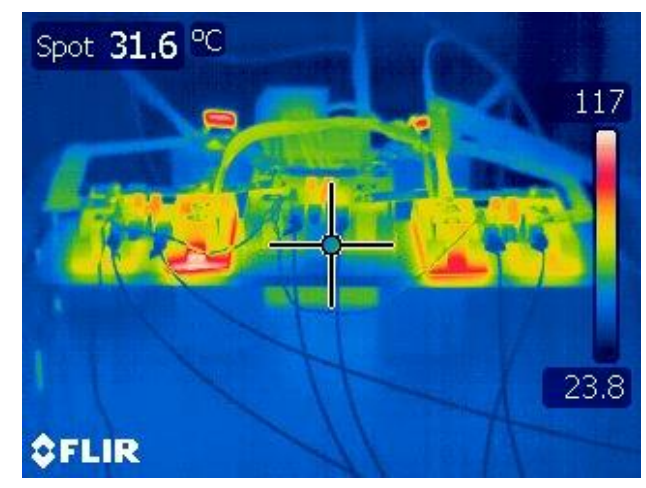

(a) Stress Out/Stress in 50\%-50\% Model Experiment

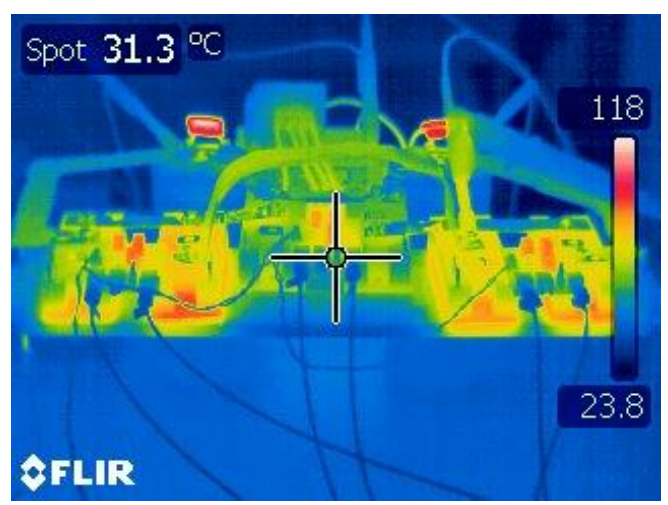

(b) Stress Out/Stress in $80 \%-20 \%$ Model Experiment

Figure 5. Losses Distribution Experiments 


\section{Conclusion}

Aiming at the topology structure of traditional NPC, there exist different work losses due to different switching devices, and the distribution of losses will be changed because of the working state of photovoltaic power generation, so the heat dissipation of high power inverter is in urgent need of solving. In this paper, an improved NPC topology is proposed, which is controlled by the control strategy, so that the switching device of the inverter has more freedom of switch, thus solving the problem of the inverter's heat dissipation. Through the experiment, we can realize the different working states by using the power adjustable PWM strategy, and the loss balance of the device can be realized by adjusting the distribution of the switching loss.

\section{References}

[1] T. M. Almeida, M. S. Piedade. High performance analog and digital PLL design [C]. Circuits and Systems, 1999. ISCAS '99. Proceedings of the 1999 IEEE International Symposium on Volume 4, May 30, 4, 394(1999)

[2] H. P. Liu, S. G. Jiang, W. Wang, D. G. Xu. The maximum power point tracking based on the double index model of PV cells [C]. Power Electronics and Motion Control Conference, 2009. IPEMC '09, 17, 2113(2009)

[3] J. Vieira, A. M. Mota. Maximum power point tracker applied in batteries charging with PV panels [C]. Industrial Electronics, 2008. ISIE 2008. IEEE International Symposium on June 30, 2, 202(2008)

[4] M. Kazmierkowski, R. Krishnan, F. Blaabjerg. Control in Power Electronics. Selected Problems [M]. Academic Press(2002)

[5] M. Ciobotaru, R. Teodorescu, F. Blaabjerg. Control of single-stage single-phase PV inverter [C]. Power Electronics and Applications, 2005 European Conference, 10(2005)

[6] Z. H. Jiang, X. D. Sun, Y. Xing, L. P. Huang. based on SSC and AW control of high frequency dual Boost digital PFC power [J]. Journal of Tsinghua University (NATURAL SCIENCE EDITION), 44, 10, 1337(2004)

[7] K. Ogura, T. Nishida, E. Hiraki, M. Nakaoka, S. Nagai. Time-sharing boost chopper cascaded dual mode single-phase sine wave inverter for solar photovoltaic power generation system [C]. Power Electronics Specialists Conference, 2004. PESC04. 2004 IEEE 35th Annual, 6, 20, 4763(2004)

[8] D. G. Holmes, T. Lipo. Pulse Width Modulation for Power Converters [M]. Principles and Practice(2003)

[9] B. Yin, R. Oruganti, S. K. Panda, et al, An output-power-control strategy for a three-phase PWM rectifier under unbalanced supply conditions [J]. IEEE Transactions on Industrial Electronics, 55, 2140(2008)

[10] X. M. Yuan, W. Merk, H.Stemmler, et al. Stationary-Frame Generalized Integrators for Current Control of Active Power Filters with Zero Steady-State Error for Current Harmonics of Concern under Unbalanced and Distorted Operating Conditions [J]. IEEE Transactions on Industry Application, 38, 523(2002)

[11] R. Teodorescu, F. Blaabjerg, M. Liserre, P. C. Loh. Proportional-resonant controllers and filters for grid-connected voltage-source converters [J]. Electric Power Applications, IEE Proceedings, 153, 5, 750(2006)

[12] R. Teodorescu, F. Blaabjerg, U. Borup. A new control structure for grid-connected LCL PV inverters with zero steady-state error and selective harmonic compensation [C]. Applied Power Electronics Conference and Exposition, APEC '04. Nineteenth Annual IEEE, 580(2004)

[13] L. Ma, X. M. Jin, Y. B. Tong, F. Tang, J. Z. Liang. Small power single-phase grid connected inverter in the ratio of resonance control [J]. Beijing Jiaotong University Journal (NATURAL SCIENCE EDITION)(2010)

[14] R. H. Middleton, G. C. Goodwin. Improved finite word length characteristics in digital control using delta operators [J]. IEEE Trans. Autom. Control, 31, 1015(1986)

[15] M. Liserre, R. Teodorescu, F. Blaabjerg. Multiple harmonics control for three-phase grid converter systems with the use of PI-RES current controller in a rotating frame [J]. Power Electronics, IEEE Transactions, 21, 3, 836(2006)

[16] F. Blaabjerg, R. Teodorescu, M. Liserre, A. V. Timbus. Overview of Control and Grid Synchronization for Distributed Power Generation Systems [J]. Industrial Electronics, IEEE Transactions, 53, 5, 1398(2006)

[17] D. Sera, R. Teodorescu, J. Hantschel, M. Knoll. Optimized Maximum Power Point Tracker for Fast-Changing Environmental Conditions [J]. Industrial Electronics, IEEE Transactions, 55, 7, 2629(2008)

[18] J. B. Hu, Y. K. He. Modeling and control of grid-connected voltage-sourced converters under 
generalized unbalanced operation conditions [J]. IEEE Transactions on Energy Conversion, 23, 3, 903(2008)

[19] F. B. Blanco, M. W. Degner, R. D. Lorenz. Dynamic analysis of current regulators for AC motors using complex vectors [J]. IEEE Transactions on Industry Applications, 35, 6, 1424(1999)

[20] H. Kim, R. D. Lorenz. A virtual translation technique to improve current regulator for salient-pole AC machines [C]. 2004 IEEE 35th Annual Power Electronics Specialists Conference, 1487(2004)

[21] F. D. Mango, M. Liserre, A. D. Aquila. Overview of Anti-Islanding Algorithms for PV Systems. Part II: Active Methods [C]. Power Electronics and Motion Control Conference, 2006. EPE-PEMC 2006. 12th International Aug. 30 2006-Sept. 1, 1884(2006)

[22] J. M. Guerrero, J. Matas, L. G. Vicuna, M. Castilla, J. Miret. Wireless-Control Strategy for Parallel Operation of Distributed-Generation Inverters [J]. Industrial Electronics, IEEE Transactions, 53, 5, 1461(2006)

[23] J. M. Guerrero, J. Matas, M. Castilla, J. Miret. Output Impedance Design of Parallel-Connected UPS Inverters With Wireless Load-Sharing Control [J]. Industrial Electronics, IEEE Transactions, 52, 4, 1126(2005)

[24] L. Ma, S. Kai, R. Teodorescu, M. Josep, X. M. Jin. An Integrated Multifunction DC/DC Converter for PV Generation Systems [C]. ISIE 2010 - IEEE International Symposium on Industrial Electronics(2010)

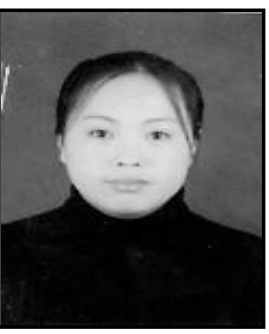

\section{Authors}

Rui Guo, received the M.S degree in Power System and Automation from Shenyang University of Industry, Shenyang, China, in 2008. She is currently a associate professor with the Academy of Renewable Energy, Shenyang Institute of Engineering, Shenyang. Her current research interests include solar photovoltaic generation technology and electrical equipment.

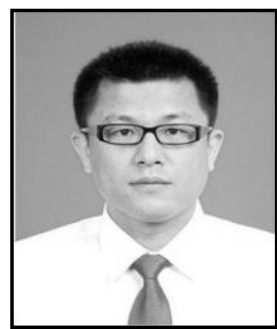

Liaoyi Ning, received the Ph.D degree of Engineering from Electric Power Engineering Department of Tsinghua University in 2010. At present he works in Economic Technology Research Institute, State Grid Liaoning Electric Power Supply Co. Ltd as the director of Programming \& Evaluation Center. Recently, his research area includes programing \& operation of electric power system, power transmission and transformation project design renewable energy generation, etc.

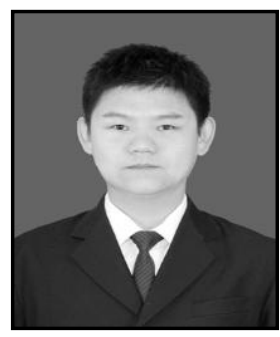

Jinyuan Liu, received the M.S degree of Management from School of Economic Management of Northeast Dianli University in 2013. At present he works in Economic Technology Research Institute, State Grid Liaoning Electric Power Supply Co. Ltd as the specialist engineer of Technology \& Economic Center. Recently, his research area includes communication technology \& economic, information science \& technology management, etc. 
International Journal of Grid and Distributed Computing

Vol. 9, No. 9 (2016) 